\title{
High-Velocity Maser Emission From Shocks Along Spiral Waves In The Circumnuclear Disk Of NGC 4258
}

\author{
Eyal Maoz \\ Astronomy Department, University of California at Berkeley, CA 94720
}

\begin{abstract}
VLBI observations of $\mathrm{H}_{2} \mathrm{O}$ maser line emission from the central sub-parsec region of the galaxy NGC 4258 have recently revealed a remarkably well-defined, rapidly rotating molecular disk which is viewed nearly edge-on. We show that the peculiar clustering of the maser sources into several distinct clumps which are almost equally spaced apart from one another, and the striking asymmetry in the high-velocity maser spectrum can be naturally explained by spiral activity in the disk.
\end{abstract}

\section{The Intriguing Regularity In The Masers' Spatial Distribution}

The circumnuclear disk in the central sub-parsec region of NGC 4258 (Miyoshi et al. 1995) is delineated by high-velocity maser sources with line-of-sight velocities that trace a Keplerian rotation curve to a remarkably high precision $(\lesssim 0.5 \%)$. This finding implies that the high-velocity sources must lie along a single diameter through the disk, presumably the one which is perpendicular to the line-of-sight (hereafter, the "midline") since this region supports the longest path-lengths for coherent amplification in our direction. Yet, the high-velocity maser sources are not distributed uniformly nor randomly along the midline, but are clustered into several distinct clumps which are almost equally spaced apart from each other - an intriguing regularity which becomes even more pronounced when including two additional, weak high-velocity maser features (Nakai et al. 1995), not reported previously.

The intriguing clustering is unlikely to arise simply due to small gas clumps in the disk, inside which the conditions for generation of maser radiation are somehow favorable, not only because of the suspicious nearly equal spacing between the clumps, but because it would imply equal chances for detecting maser emission from clumps at any location in the disk - a situation which is firmly ruled out by the nearly perfect Keplerian rotation curve.

The intriguing maser distribution can be naturally explained by the existence of spiral structure in the disk (Maoz 1995). The maser sources appear clustered at the intersections of spiral arms and the diameter through the disk of maximal gain path, and are thus spaced apart at the characteristic crestto-crest radial distance between the spirals. It is interesting to notice that the estimated parameters of the disk indeed support the existence of spiral structure with the right inter-arm separation, whose origin lies with the local gravitational instability picture (e.g. Goldreich \& Lynden-Bell 1965). Spiral pattern appears when the local Toomre Q-parameter $Q \equiv c_{s} \kappa /(\pi G \Sigma)$ satisfies $1 \lesssim Q \lesssim 2$, and is most pronounced at a radial crest-to-crest separation given by $\lambda_{\text {crit }} \simeq 4 \pi^{2} G \Sigma / \kappa^{2}$, 
where $c_{s}$ is the sound speed, $\kappa=\Omega(r)$ is the epicyclic frequency in a Keplerian disk, and $\Sigma$ is the surface mass density. Assuming the disk mass is dominated by molecular gas of density $n$, substituting $\Sigma=n h$ where $h$ is the disk thickness, requiring that the disk maintains spiral activity with $\lambda_{\text {crit }} \simeq 0.75$ mas (the mean inter-clump separation), and assuming an hydrostatic equilibrium $h / r=c_{s} / \Omega r$, we obtain molecular density of $n_{\mathrm{H}_{2}} \simeq 1.8 \times 10^{10}(Q / 1.6)^{-1} \mathrm{~cm}^{-3}$, and disk thickness of $h \simeq 0.1(Q / 1.6)$ mas, in nice agreement with independent estimates by Miyoshi et al. (1995) of $n_{\mathrm{H}_{2}} \approx 10^{10} \mathrm{~cm}^{-3}$ and $h \lesssim 0.1$ mas.

\section{The Doppler-Shift Asymmetry}

While the fact that the red-shifted and blue-shifted high-velocity masers fit into the same Keplerian relation indicates the disk is very nearly circular, a striking asymmetry appears in the maser spectrum: the red-shifted high-velocity sources are much more numerous and more intense than the blue-shifted ones. The same strong asymmetry appears also in the masing circumnuclear disk in NGC 1068 (Greenhill et al. 1996), suggesting that the relatively enhanced red-shifted maser emission may be a general phenomenon. Herrnstein et al. (1996) suggested that the asymmetry in NGC 4258 may be attributed to the warp in the disk. Although plausible, such explanation relies on a coincidentally special viewing angle of the disk, which is somewhat improbable, especially in light of the similar finding in NGC 1068, and the absence of a counter example. We now argue that the observed Doppler-Shift asymmetry can naturally arise due to shocks along spiral waves in circumnuclear disks (Maoz \& McKee 1996).

A collisional pump mechanism of the maser transition is characterized by the emission of infrared line photons which must not be re-absorbed by the molecules if inversion is to be maintained. Based on detailed numerical calculations of IR photon trapping (e.g. Hollenbach, Elitzur \& McKee 1996), and assuming low optical depth in cold dust, we find that in disks of thickness $\sim 10^{16} \mathrm{~cm}$ and molecule density $\sim 10^{9-10} \mathrm{~cm}^{-3}$, as estimated for the circumnuclear disk in NGC 4258, the level populations of the maser transition are likely not to be inverted in most of the disk volume, except perhaps within two thin slabs of thickness $\sim 10^{13} / n_{9} \mathrm{~cm}$ in the upper disk atmosphere. This means that maser radiation passing through ambient molecular gas which maintains close velocity coherence with the maser source is absorbed in the disk.

Shock origin of interstellar $\mathrm{H}_{2} \mathrm{O}$ masers has been previously suggested by many authors. Detailed modeling revealed that the physical and chemical conditions in post-shock regions are favorable for the generation of luminous 22 $\mathrm{GHz}$ maser emission by collisional pumping: the energy to pump the masers is provided by the dissipation of the kinetic energy in the shock, and the chemical reactions in the warm, dense post-shock gas lead to the production of copious amounts of warm $\mathrm{H}_{2} \mathrm{O}$, both in the case of J-type $\left(v_{s} \gtrsim 30 \mathrm{~km} \mathrm{~s}^{-1}\right)$ shocks as well as in non-dissociative MHD, C-type shocks $\left(10 \lesssim v_{s} \lesssim 40-50 \mathrm{~km} \mathrm{~s}^{-1}\right)$. Spiral shocks may develop from disturbances induced at the outer edge of the disk that steepen into shocks as they propagate inward (e.g. Spruit 1987), or due to slamming of the orbiting gas as it runs into the more slowly rotating spiral waves, as suggested in the case of spiral shocks in galactic disks (e.g. Roberts 1969). 
Under quite a wide range of shock scenarios, maser action takes place within a column density of $\sim 10^{22-23} \mathrm{~cm}^{-2}$ behind the shock front, i.e. in a slab of thickness $\sim 10^{13-14} / n_{9} \mathrm{~cm}$. The sheet-like geometry of the shocks, combined with the relative velocity between the post-shock and pre-shock gas ensures that the IR photons could escape freely through the shock front, thus avoiding quenching the maser at any depth into the disk. Since maser intensity strongly depends on the length along the l.o.s through the masing slab, the strongest and thus most likely detectable high-velocity masers appear where the l.o.s is tangent to an arc.

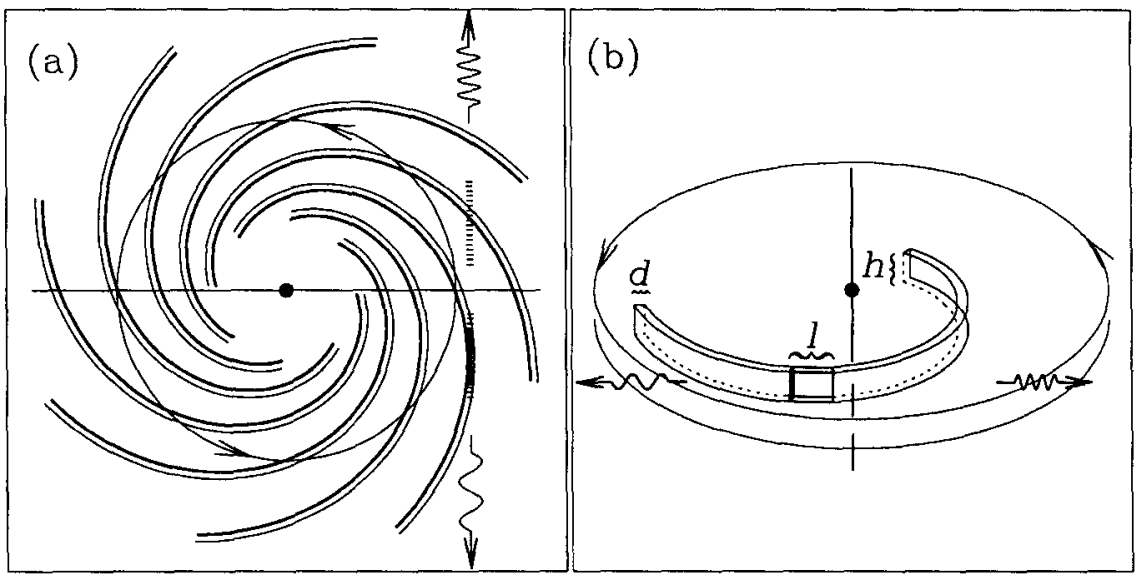

Figure 1: Locations of high-velocity masers that originate along spiral shocks.

The general geometry is illustrated in Figure 1-a where each spiral region represents a masing slab behind a shock front. The horizontal line is the midline - the diameter through the disk which is perpendicular to the l.o.s. The narrow, dark rectangular denotes the location of one of the high-velocity masers. The maser emission comes from box-shaped regions of thickness $d$, length $l \simeq(8 r d)^{1 / 2}$ and height $h$ (Fig.1-b), where in NGC $4258 d \ll h \lessgtr l$. Since spiral shocks have trailing geometry due to the action of differential rotation, we see that the highvelocity masers are always located on the front side of the midline in the receding side of the disk, and on the rear side of the midline in the approaching side of the disk (Fig.1-a). Since the interaction of maser photons with $\mathrm{H}_{2} \mathrm{O}$ molecules is a line process, and the l.o.s velocity is symmetric about the midline, blue-shifted maser photons are subject to absorption as they pass through the velocitycoherent region on the other side of the midline (denoted by the shaded region in Fig. 1-a). The length of that absorbing column of gas $\lambda$ is determined by velocity coherence and is calculated based on the velocity field in the disk. In general, $\lambda$ can be effectively reduced due to the finite disk inclination, the more so in a thin disk. In the case of NGC 4258, where the pitch angle of the spiral shocks is small $\theta_{p} \approx v_{s} / r(\omega-\Omega) \sim 1-3^{\circ}$, where $v_{s}$ is the shock speed (the flow velocity normal to the spiral in a frame corotating with the wave), we obtain $\lambda \simeq 10^{16.5-17} \mathrm{~cm}$. The shocks are unlikely to be strong since they would otherwise lead to a rapid radial transfer of mass. 
The absorption coefficient is derived assuming the gas is at LTE. This is a rough approximation, partly because the exposure to very intense maser radiation may perturb the level populations in the absorbing region. At the expected low gas temperatures of $\approx 100 \mathrm{~K}$ (lower limit of $\approx 70 \mathrm{~K}$ is set by the $\mathrm{X}$-ray irradiation of the disk by the central source), we obtain an excess optical depth for the blue-shifted emission of

$$
\tau\left(5_{2,3}-6_{1,6}\right) \simeq 13 n_{9}\left(\mathrm{H}_{2}\right) \lambda_{16} x_{-4}\left(\mathrm{H}_{2} \mathrm{O}\right) T_{2}^{-2} \exp \left[6.42\left(1-T_{2}^{-1}\right)\right]
$$

where $\lambda_{16} \equiv \lambda / 10^{16} \mathrm{~cm}, T_{2} \equiv T / 100 \mathrm{~K}$. Stimulated emission was taken into account. Given the estimated properties of the disk in NGC 4258, a significant absorption of blue-shifted high-velocity maser emission is expected, which would result in asymmetric maser spectrum, as observed.

The high-velocity masers thus do not arise along the midline, but rather where the column density of masing gas is maximal - where the l.o.s is tangent to a spiral. If the spirals have similar pitch angles (or in the case of a single winding spiral with constant $\theta_{p}$ ), the masers are located along a diameter through the disk which makes an angle $\theta_{p}$ with the midline. In such case, and in the case of small pitch angles as in NGC 4258, there would still be no noticeable deviation from a Keplerian rotation curve. We should briefly comment that the lowvelocity features are unaffected since they arise due to amplification of a central background continuum source by masing gas inner to the quenched zone.

\section{Conclusion}

We argue that the high-velocity masers observed in NGC 4258 arise in thin, spiral-shaped slabs of masing gas, along shock fronts in a largely non-masing disk. It can naturally explain the observed regularity in the distribution of the maser sources. The Doppler-shift asymmetry is due to the trailing geometry of the shocks, which makes the blue-shifted high-velocity maser photons pass through a velocity-coherent region of non-inverted gas along the l.o.s to an observer, where they are absorbed. The estimated degree of absorption can easily produce a noticeable asymmetry in the maser spectra of circumnuclear disks with wide range of parameters, including the one in NGC 4258.

\section{References}

Goldreich , P. \&Lynden-Bell, D. 1964, MNRAS, 130, 125

Greenhill, L.J. et al. 1996, Submitted to the ApJ

Hollenbach, D.J., Elitzur, M. \&McKee, C.F. 1996, in preparation.

Maoz, E. 1995, ApJ, 455, L131

Maoz, E., and McKee, C.F. 1996, submitted to ApJ

Miyoshi, M. et al. 1995, Nature, 373, 127

Nakai, N. et al. 1995, PASJ, 47, 771

Roberts, W.W. 1969, ApJ, 158, 123

Spruit, H.C. 1987, A\&A, 184, 173 